\title{
REALIDAD MENTAL EN LA CIENCIA COGNITIVA
}

\author{
MENTAL REALITY IN COGNITIVE CIENCE \\ NicolÁs MEdinA \\ Universidad Nacional Mayor de San Marcos, Lima, Perú \\ (RECiBido El 04/01/2010, ACEPTADO EL 01/06/2010)
}

\begin{abstract}
RESUMEN
El objetivo de este trabajo es demostrar el carácter objetivo del fenómeno mental, describiendo las propiedades naturales que le confieren poderes causales que impactan efectivamente en nuestro organismo físico, produciendo conductas. Las argumentaciones de este trabajo siguen una orientación cognitiva que reconoce la mente como una entidad representacional simbólica y computacional, defendida por Jerry Fodor.

Palabras clave: interacción mente-cuerpo; causalidad mental; intencionalidad; mente representacional; representación simbólica-computacional; actitudes proposicionales; lenguaje del pensamiento; máquina de Turing; tesis de Church-Turing.
\end{abstract}

\begin{abstract}
The aim of this paper is to show the objective nature of the mind describing their natural properties that should have causal powers to make an impact on physicals organisms. Arguments of this paper follow the cognitive view that recognize the mind as a symbolic representational and computational entity, defended by Jerry Fodor.
\end{abstract}

Keywords: mind-body interaction; mental causality; intentionality; representational mind; symbolic-computational representation; propositional attitudes; Turing machine; ChurchTuring thesis.

1 Docente Principal de la Facultad de Psicología de la UNMSM.

E-mail: medina0712@yahoo.com 


\section{INTRODUCCIÓN}

Este es un trabajo enmarcado en el dominio filosófico de la psicología, que tiene como objetivo exponer las ideas centrales que confieren objetividad al estudio de la mente y sobre esa base analizar las posibilidades explicativas de la mente desde una perspectiva causalista. En otras palabras, el propósito principal de este artículo es analizar las propiedades naturalizables de la mente que lo caracterizaría como un sistema mental con mecanismos reales y objetivos, y que le proporcionaría poder causal para dar explicaciones científicas (nomológicas) del comportamiento humano.

Como es sabido, la mente es considerada como un fenómeno complejo de naturaleza no material, no física, que dificulta la explicación cognitiva que los estados mentales pueden generar impacto causal sobre el organismo físico para generar ciertas conductas. $\mathrm{Al}$ respecto, algunos filósofos materialistas sostienen que las entidades o eventos materiales que poseen propiedades físicas pueden ser causa de otras entidades o eventos materiales portadoras de propiedades físicas. Es decir, la causalidad solo es efectiva en entidades materiales físicas y no en entidades inmateriales, intangibles, como la mente. De modo que, para algunos materialistas, hablar de relación causal entre una entidad no material (por decir, la mente) y otra material (por decir, cuerpo-conducta) es un absurdo. En consecuencia, la naturaleza no material de la mente hace difícil explicar cómo los estados mentales pueden influir causalmente en la producción de conductas (físicas).

Ese problema está asociado al problema de la interacción mente-cuerpo, tratado de manera sistemática por Descartes, quien sostuvo que la mente y el cuerpo en el ser humano son dos sustancias de distinta naturaleza que interactúan, en el sentido de que el pensamiento (o mente) es la condición primaria que causa los diversos modos de comportamiento físico en el ser humano, exceptuando aquellas conductas de naturaleza refleja (los actos reflejos). La solución cartesiana al problema de la conexión mente-cuerpo fue formulada a través de la hipótesis de la existencia de la "glándula pineal" (ubicada en la base cerebral) que actuaría como un homúnculo que cumplía la función de vincular el pensamiento con el cerebro. Esa hipótesis no satisfizo las exigencias teóricas y metodológicas de las ciencias cognitivas actuales.

Tradicionalmente, en el dominio de la filosofía de la mente se han observado dos grandes teorías que han intentado resolver el problema de la interacción mente-cuerpo, así tenemos: la teoría dualista que considera la mente como una sustancia no física, y la teoría materialista que sostiene que la mente no es de naturaleza distinta de lo físico, o sea, todos los estados, propiedades, procesos y operaciones mentales son en principio idénticos con los estados, propiedades, procesos y operaciones físicas.

\section{ALTERNATIVAS FISICALISTAS AL PROBLEMA MENTE-CUERPO}

El dualismo cartesiano expresado en el problema de la interacción mente-cuerpo, tuvo gran impacto en la filosofía y la psicología, generando múltiples reacciones con planteamientos alternativos al dualismo, entre las que se cuentan la concepción conductista, con sus dos versiones: el conductismo lógico y el conductismo psicológico, y la concepción reduccionista representada por la teoría de la identidad y el materialismo eliminativista. 


\section{Conductismo lógico}

El conductismo lógico está asociado con las ideas de Gilbert Ryle, Rudolf Carnap (representante del positivismo lógico) y Ludwig Wittgenstein, que postularon una teoría semántica sobre el significado de los términos mentales. En su libro The concept of mind (El concepto de lo mental), Ryle (1949) sostuvo que es un error hablar de lo mental como una categoría abstracta que refiere a propiedades internas de un agente que causa comportamientos. En vez de lo mental, lo que realmente existe son las disposiciones para la conducta, a las que equivocadamente se las ha llamado "mente". Según Ryle, el lenguaje mental es una manera de hablar sobre las disposiciones para comportarse y constituye el patrón de la conducta humana. La mente no actúa como un "fantasma en la máquina" corporal, sino que es la suma de las disposiciones conductuales. Por ejemplo, cuando se atribuye un estado mental de sed a un organismo, es lo mismo que decir que el organismo está dispuesto a conducirse de una manera particular, como la de beber si hay agua disponible. En ese sentido, toda atribución mental es equivalente en significado a una afirmación de la forma "si-entonces" (llamado conducta hipotética) que expresa una disposición conductual. Precisando el ejemplo anterior, cuando decimos "David está sediento" tendría un significado equivalente a la afirmación disposicional "Si hubiera agua disponible, entonces David la bebería". Como se puede observar, en la definición de una conducta hipotética no se incluyen términos mentales, ya que la cláusula si de la conducta hipotética habla solo de estímulo y la cláusula entonces habla solo de respuestas conductuales. En virtud de que los estímulos y respuestas son eventos físicos, el conductismo lógico es una especie de materialismo. En suma, se puede decir que el conductismo lógico es una postura filosófica que sostiene que toda atribución mental puede ser traducida a una conducta hipotética única, o sea, a disposiciones conductuales físicas.

La posición conductista lógica de Carnap $(1932,1933,1978)$ tiene una orientación epistemológica, asumiendo que es posible estudiar científicamente los fenómenos psicológicos considerándolos no como vivencias subjetivas, sino como expresiones proposicionales que hacen referencia a conductas físicas. Carnap, concordante con su concepción positivista lógica de la ciencia, reconoce la distinción entre enunciados teóricos y enunciados observacionales (o protocolares), los que, mediante las "reglas de correspondencia" aplicadas a la psicología, pueden traducirse de términos teóricos (que refieren a estados mentales) a términos observacionales (que hacen referencia a conductas físicas que acompañan a los estados mentales). Por ejemplo, es posible fisicalizar la proposición teórica "Juan está excitado", a través de las proposiciones observacionales: "a Juan le tiemblan las manos", "a Juan le tiembla la voz", "Juan tiene taquicardia”, entre otros enunciados que hacen referencia a respuestas físicas del organismo de Juan. Dicha traducción facilita la relación directa de los términos observacionales con los referentes físicos conductuales que finalmente determinarán el carácter verdadero y el significado empírico de esas proposiciones. Mientras que las proposiciones teóricas (conceptuales) no presentan un referente empírico directo, lo cual dificulta el establecimiento de su significado empírico y su carácter verdadero. En consecuencia, la postura positivista lógica de Carnap apunta a fisicalizar la psicología (de los estados mentales) a través de enunciados observacionales que refieren conductas físicas. 
Por su lado, Wittgenstein $(1953,1988)$ vinculó los estados mentales subjetivos con los lenguajes privados. Según este autor, un lenguaje privado es un lenguaje cuyas palabras se refieren a lo que solo puede ser conocido por la persona que habla, o sea, a sus sensaciones privadas inmediatas. Este lenguaje no podría ser entendido por otra persona porque estaría expresando la vivencia personal privada de la persona que habla. Wittgenstein cuestionó la utilidad semántica y pragmática del lenguaje privado, y consideró confiable el lenguaje público que tiene referente ostensivo (como conductas) que pueden ser conocidos por otras personas. Sin embargo, Wittgenstein consideró que un estado mental como el "dolor" puede expresarse en conductas (como ocurre realmente de manera natural) u otras expresiones corporales, haciendo pública la sensación de dolor. Aquí se pondría en práctica el "juego del lenguaje" con la palabra "dolor", que consiste en que la gente que lo juega adopte un comportamiento de la forma concreta que llamamos expresión de dolor. En esa perspectiva, la posición de Wittgenstein se diferencia del conductismo al objetar la siguiente idea: si el dolor se identifica con la conducta de dolor, entonces cuando se dice "A tiene dolor" significa que "A se está conduciendo de tal y tal manera". El cuestionamiento de Wittgenstein a esa interpretación conductista de dolor va dirigido en el siguiente sentido: el lenguaje de dolor está conectado (no identificado) con la conducta de dolor, de modo que "dolor" no significa llanto: la expresión verbal de dolor no describe la expresión natural de dolor, sino que ocupa su lugar. Entonces se observa que el dolor y la conducta de dolor son separables, pero su conexión se da a través de las conductas ostensivas que expresan dolor, las que pueden enunciarse mediante un lenguaje público.

Según Wittgenstein, las palabras que usamos para designar sensaciones y que están vinculadas a nuestras expresiones naturales de sensación, son aprendidas en conexión con la expresión natural. Por eso, se puede decir que el aprendizaje del significado de las palabras que refieren estados privados (mentales) de las personas se basarían en el criterio de intersubjetividad (o acuerdo colectivo convencional), mediante el cual se otorgaría carácter público al lenguaje privado de las personas, confiriéndole objetividad y significatividad.

\section{Conductismo psicológico}

El conductismo psicológico en sus dos versiones (conductismo clásico, representado por J. Watson, y conductismo operante, representado por B. F. Skinner) propugnó la idea de que la conducta humana no está determinada causalmente por la mente, sino por estímulos del medio ambiente. Skinner se ocupó de catalogar las leyes que determinan las relaciones causales entre estímulos y respuestas. En esta concepción conductista se propone eliminar el problema de la interacción mente-cuerpo, señalando la no existencia de tal problema.

\section{Reduccionismo}

La concepción reduccionista constituye una alternativa filosófica frente al dualismo y al conductismo, alegando la reconceptualización de lo mental a través de la teoría de la identidad, cuya tesis nuclear es que los estados y procesos mentales son estados y procesos del cerebro. En tal sentido, los estados mentales son idénticos a los estados cerebrales. La 
teoría de la identidad se basó, por un lado, en la concepción monista de la realidad (que está constituida de materia física) y, por otro lado, en una concepción metodológica que defendió la reducción del lenguaje mentalista a un lenguaje físico, acorde con el lenguaje de las ciencias físicas.

Esa orientación reduccionista es la que también postula el materialismo eliminativista que sostiene que la psicología de sentido común (que trata sobre creencias, deseos, pensamientos) no describe nada, por lo tanto debería ser eliminada. Los eliminativistas consideran que la neurofisiología es la encargada de elaborar las teorías científicas sobre las funciones psíquicas. Al respecto, Churchland $(1984,1999)$ caracterizó la concepción materialista eliminativo en los siguientes términos:

[...] No se podrá efectuar una reducción interteórica del marco de referencia psicológico corriente, porque el marco de referencia psicológico que utilizamos corrientemente es una concepción falsa y radicalmente engañosa sobre las causas de la conducta humana y la naturaleza de la actividad cognitiva. Desde esta perspectiva, la psicología habitual no solamente constituye una representación incompleta de nuestra naturaleza interna, sino directamente constituye una mala representación de nuestros estados y actividades internas. En consecuencia, no es posible esperar que una explicación neurocientífica verdaderamente adecuada de nuestra vida interior proporcione las categorías teóricas que se corresponden escrupulosamente con las categorías de nuestro marco de referencia habitual. Consecuentemente, lo único que se debe esperar es que el antiguo marco simplemente sea eliminado y no que pueda reducirse por una neurociencia más desarrollada (Churchland, 1984, 1999; págs. 75-76).

En ese sentido, el materialismo eliminativista está de acuerdo con que la neurociencia sería la encargada de realizar el estudio científico de la actividad cognitiva, y no la psicología de sentido común (folk psychology), que debería ser eliminada por la vacuidad de sus teorías. (Aquí no vamos a sustentar la tarea primordial que pretende la psicología de sentido común para el desarrollo de la psicología cognitiva, que merece ser tratado en otro lugar).

\section{ALTERNATIVAS COGNITIVISTAS AL PROBLEMA MENTE-CUERPO}

En concordancia con el propósito del presente trabajo que está dirigido a destacar el papel importante que cumple la actividad mental en la explicación causal del comportamiento humano, vamos a exponer las principales ideas de Davidson, Putnam y Fodor, en relación con la solución al problema de la interacción mente-cuerpo.

Donald Davidson describió la naturaleza causal de la mente en un trabajo titulado Eventos Mentales, publicado en 1970, en el que afirmó que los eventos mentales se basan en tres principios: 1) algunos eventos mentales (como la percepción, recuerdo, decisión) interactúan causalmente con eventos físicos (principio de interacción causal). Por ejemplo, si alguien viajó en avión para ir a otro país, previamente han debido ocurrir varios eventos mentales, como informaciones, juicios, cálculos, decisiones, acciones intencionales y cambios de creencias, que jugaron un papel causal en el hecho de viajar. Según el principio de interacción causal de Davidson, la persona que tomó el avión para viajar a otro país movió su cuerpo en una forma que fue causada por eventos mentales de cierto tipo, y este 
movimiento corporal, a su vez, causó el hecho de tomar el avión para viajar; 2) donde hay causalidad debe haber una ley, es decir, los eventos relacionados como causa y efecto están bajo estrictas leyes deterministas (principio de carácter nomológico de la causalidad), y 3) no hay estrictas leyes deterministas sobre la base de que los eventos mentales pueden ser predecidos y explicados (anomalía mental). En suma, estos principios constituyeron el sustento de la relación mente-cuerpo postulada por Davidson: las acciones libres del hombre son explicadas mediante los deseos, conocimientos y percepciones (ver David M. Rosenthal (ed.). The Nature of Mind; 1991, págs. 247-256).

Por su lado, Hilary Putnam publicó en 1975 su Philosophy and Our Mental Life, en el que expone su concepción sobre la mente humana, señalando de modo general que la vida mental del ser humano es un rasgo real y autónomo de nuestro mundo. Putnam caracteriza la mente apelando al concepto de isomorfismo funcional que, en breve, consiste en que dos sistemas son funcionalmente isomórficos si hay una correspondencia entre los estados de uno y los estados del otro, preservando la relación funcional. Esos dos sistemas pueden tener diferentes constituciones, pero pueden ser funcionalmente isomórficos. Por ejemplo, una computadora hecha de componentes eléctricos puede ser isomórfica a otra hecha de ruedas con dientes y otros engranajes mecánicos. O sea, para cada estado en la primera computadora existe un correspondiente estado en la otra, y las relaciones secuenciales son las mismas (si el estado $\mathrm{S}$ es seguido por el estado B en el caso de la computadora electrónica, el estado A sería seguido por el estado B en el caso de la computadora hecha de rueda dentada y otros engranajes mecánicos, y para ello no interesa que las realizaciones físicas de esos estados sean diferentes). De esa manera, una computadora fabricada con componentes electrónicos puede ser funcionalmente isomórfica a una fabricada con ruedas dentadas y otros engranajes mecánicos, o puede ser funcionalmente isomórfica a empleados de escritorio usando lápiz y papel. En ellos la constitución física es diferente, pero el aspecto funcional es el mismo. En esa perspectiva, el nivel físico del cerebro puede realizar una función que puede ser isomórfica a la función realizada por la mente. De modo que la mente interacciona con el cerebro en el nivel funcional.

Además, Putnam aclaró que la funcionalidad física del cerebro está regida por leyes propias de su naturaleza, que pertenecen al nivel neurofisiológico y/o neuroquímico, que lo distinguen de las leyes que rigen los mecanismos mentales. Así, la mentalidad del ser humano se constituye en un nivel autónomo de nuestro mundo. Putnam (1975) también observó que la cuestión de cuál es la naturaleza de nuestra sustancia (¿es espiritual, o es material, o somos de queso suizo?) no es lo más importante, sino que lo más significativo es analizar ¿cuál es nuestra forma intelectual?, y no qué es la materia. En suma, Putnam concluyó que "tenemos lo que siempre quisimos" ("we have what we always wanted"), con lo que caracteriza la autonomía y realidad de la vida mental, sin recurrir a entidades misteriosas, ni a agentes fantasmales, ni al élan vital, como causas para tener dicha autonomía mental (Putnam, 1975; pág. 99).

En cuanto al punto de vista de Jerry Fodor sobre la interacción mente-cuerpo, se puede decir que él está convencido de que la mente juega un papel significativo en la causación de conductas. Este presupuesto de Fodor se sustenta en tres ideas esenciales que caracterizan la naturaleza de la mente: el funcionalismo, la intencionalidad y la representación mental. 
Respecto al funcionalismo, Fodor aceptó en gran parte la tesis propuesta por Putnam (1975), manteniendo el supuesto que los estados mentales pueden materializarse en sistemas y entidades físicos, sin riesgo de dar explicaciones físicas, ya que dicha materialización se realizaría sobre base del principio de isomorfismo funcional. El tono intencionalista de Fodor se expresa en la caracterización de la mente como un sistema representacional, constituido por estados mentales en la forma de actitudes proposicionales que vienen a ser tendencias para expresar lingüísticamente ciertos propósitos. Las actitudes proposicionales representan creencias, deseos, temores, motivaciones, etc.

En relación a la representación, Fodor consideró que los procesos cognitivos que poseen carácter representacional son computacionales en la medida que la característica representacional se basa en símbolos que permiten su manipulación. Es decir, la principal característica de la mente es que es un sistema representacional simbólico (de tipo lingüístico), dentro del cual tiene lugar el pensar (que se expresa mediante computaciones simbólicas). Por eso, Fodor $(1975,1984)$ sostuvo la hipótesis del lenguaje del pensamiento, que es una idea sobre la forma en que nos representamos el mundo y una idea sobre cómo ha de ser explicada nuestra conducta. En tal sentido, Fodor sostuvo que nosotros como agente generamos nuestras conductas debido a que disponemos de un sistema representacional, y el hecho de decidir nuestras conductas es un proceso computacional. En otros términos, el acto que realizamos es consecuencia de computaciones definidas sobre las representaciones de las posibles acciones. De modo que, "[...] si no hay representaciones, no hay computaciones" (Fodor, 1975, 1984; pág. 51). Al mismo tiempo Fodor aclara que el lenguaje del pensamiento no es simplemente un medio de manipulación de símbolos (o sea, no solo pensamos), sino que pensamos acerca de ciertas cosas, y que sin duda esas cosas existen en el mundo. Eso quiere decir que el lenguaje del pensamiento es un "mentalés" lingüístico (sistema representacional) que da sentido a las cosas del mundo. En suma, se puede decir que Fodor tiene el convencimiento de que la mente tiene una existencia real y que es posible estudiarla científicamente en colaboración con la metodología empleada por la psicología cognitiva, la lingüística y otras ciencias cognitivas.

\section{CAUSALIDAD MENTAL}

Responder afirmativamente a la pregunta: ¿la mente tiene efectos en el mundo físico? es creer en la causalidad mental. Con la adopción de esta postura se puede explicar que las acciones de las personas están causadas por sus pensamientos, creencias, intenciones, deseos y otras actitudes proposicionales. Por ejemplo, cuando decimos que María ingirió una aspirina porque ella pensó que le disminuiría el dolor de cabeza, en este caso se observa una causación mental al decir "porque", que expresa una relación causal entre el pensar de María y su acción, así como podría ocurrir en casos físicos, no mentales, como cuando decimos que el vidrio de una ventana se quebró porque un ladrillo lo impactó. En esos casos, se puede decir que tanto el pensar de María como el impacto del ladrillo, son causalmente eficaces. De modo que, siempre que hacemos algo o pensamos algo porque algo está ocurriendo en nuestras mentes, eso es un caso de causación mental.

¿En qué consiste una causación mental? Al respecto, Crane (s/d) sostuvo que la causación mental es cuando un estado mental (como una creencia o intención) o un evento mental 
(como una experiencia) tiene un efecto, que puede ser o mental (como otro pensamiento o experiencia) o físico (como un efecto en el mundo físico). En cualquier caso de causación se pueden distinguir entre la conexión de causación (lo que está siendo relacionado) y la relación misma. Así, por ejemplo, cuando el ladrillo causó la rotura del vidrio se pueden distinguir entre la causa (impacto del ladrillo), el efecto (rotura del vidrio) y la relación misma (causación) que vinculan esos dos eventos. En ese sentido, se dice que hay causación mental cuando por lo menos uno de los elementos de la relación, de alguna interacción causal, es mental, y se dice que hay causación física cuando se observa que por lo menos uno de los elementos de la relación, de alguna interacción causal, es físico.

Consecuentemente, se puede ver que la causación mental es un hecho real, innegable, dada la obviedad de un fenómeno de causación mental como lo muestra el siguiente caso: supongamos que usted ve a un amigo y esto causa que le haga un gesto, ¿cómo? Puede ocurrir algo como lo siguiente: la luz es reflejada desde la figura real del amigo sobre su retina; los impulsos viajan a través de su nervio óptico; luego, su córtex estriado procesa la información llevada por el nervio óptico; usted forma (de algún modo) la creencia de que su amigo está allí; esto hace que usted tenga la intención de saludar a su amigo; lo que produce que ciertas cosas ocurran en su sistema motor; ellos causan el acto de levantar la mano. En este caso, los hechos físicos y mentales están involucrados en la cadena de causación, en la que, obviamente, el acto de levantar la mano como gesto de saludo (hecho físico) ha sido causado por la creencia de la presencia del amigo y por la intención de saludarle (hecho mental).

\section{Intencionalidad (aboutness)}

En el problema de la causación mental se presenta otra cuestión referida a la intencionalidad. Según Lycan $(1999,2002)$, el término intencional es utilizado por los filósofos en el sentido de "dirigido sobre un objeto". Coloquialmente se puede decir que una entidad es intencional en la medida que es (o versa) acerca de algo. Por ejemplo, las creencias, los deseos y los pesares son acerca de algo, o sea, tienen "objetos intencionales": tengo creencias acerca de Nelson Mandela; quiero que haya paz en el mundo; me lamento de haber faltado a una reunión importante. Un aspecto problemático de la intencionalidad es su indiferencia a la realidad. El objeto intencional no necesita existir o estar dado, de modo que los griegos rendían culto a Zeus; un amigo mío cree que los cerditos vuelan. Al respecto, Crane (1992) formuló la siguiente cuestión: ¿cómo puede causar cualquier efecto un estado intencional (mental) que versa sobre algo abstracto, sin referente empírico? En relación a ello, Lycan $(1999,2002)$ recordó que la intencionalidad en el fondo es representación mental, y las actitudes proposicionales tienen la propiedad que señaló Brentano: los estados y eventos mentales representan estados de cosas reales o posibles. La evidencia de que esto es así lo proporciona la relación que existe entre las propiedades intencionales y las propiedades semánticas, por eso la representación no sería un caso controvertido, ya que las creencias (como representación) pueden ser verdaderas o falsas, y al fin son creencias.

Lycan $(1999,2002)$ refiere la siguiente idea de Brentano, que caracteriza adecuadamente la naturaleza de la intencionalidad de la siguiente manera: la propiedad de ser neutral respecto a la realidad hace de la intencionalidad el rasgo distintivo de lo mental, dado que todos 
y cada uno de los objetos mentales y solo ellos son intencionales en ese sentido (Lycan, 1999, 2002; pág. 657). De modo que las creencias (como representaciones mentales) estarían compuestas por conceptos y su verdad depende de la conjunción de sus estructuras internas y de cómo es el mundo; entonces, es natural considerar su "versar sobre algo" (aboutness) como una cuestión de referencia o designación mental. Fodor (1975, 1981a) afirmó que los estados intencionales son estados físicos que tienen propiedades semánticas y que los estados de cosas existentes o inexistentes que son sus objetos no son más que contenidos representacionales. Esta idea fodoriana de que un estado mental tiene contenido intencional debido a que posee ciertas propiedades semánticas lo ejemplifica de la siguiente manera: para que Enrique crea que Galileo fue italiano, aparentemente ello implica tres formas de relaciones, que son: Enrique, una creencia y una proposición que es el contenido de la creencia (o sea, la proposición de que Galileo fue italiano). La propiedad esencial de la creencia de Enrique es que versa sobre (aboutness) Galileo (y no de otra persona, por decir, Newton) y que es verdadera "si y solo si" Galileo fue en realidad italiano. Asimismo, Fodor (1981b) sostuvo que entre los filósofos hay acuerdo en considerar que las creencias implican propiedades semánticas que se expresan en una proposición que puede ser verdadera o falsa, y que son sobre (o refieren a) una cosa antes que a otra. Esas propiedades que caracterizan al contenido intencional son representadas por el símbolo que hace referencia (o es sobre) cosas; de modo que si alguien dice "Galileo fue italiano", de acuerdo con la creencia de Enrique, esta declaración expresa una proposición sobre Galileo, que es verdadera o falsa, dependiendo del país donde nació Galileo.

Así, Fodor (1981) asume que existen tales cosas como los símbolos mentales (representaciones mentales) que poseen propiedades semánticas, y basado en ello sostuvo que el hecho de tener una creencia implica estar relacionado con un símbolo mental; también afirmó que la creencia hereda sus propiedades semánticas del símbolo mental que figura en la relación (cabe mencionar que Fodor reconoció que las creencias de un agente son determinadas por el carácter de sus experiencias y sus dotes genéticas, según las teorías del aprendizaje y la percepción, y destacó la idea de que esos estados mentales [las creencias] por sí mismos no proveen la comprensión requerida, o el sentido de las cosas o ideas). Y, a la vez, las propiedades semánticas de las palabras y oraciones que expresamos son de nuevo heredados de las propiedades semánticas de los estados mentales que expresa el lenguaje. En consecuencia, los estados mentales (actitudes proposicionales) actuarían como condiciones iniciales que causan el comportamiento humano (previa intencionalidad).

\section{Computación simbólica}

Fodor (1981) sostuvo que la asociación de las propiedades semánticas de los estados mentales con aquellas de los símbolos mentales es totalmente compatible con la metáfora de la computadora porque es natural considerar a la computadora como un mecanismo que manipula símbolos. Una computación es una cadena causal de estados de la computadora y los vínculos en la cadena son operaciones sobre fórmulas semánticamente interpretadas en un código de la máquina. Considerar un sistema como una computadora es formular cuestiones sobre la naturaleza del código sobre el que se computa y sobre las propiedades semánticas de los símbolos en el código. Ciertamente, la analogía entre 
mentes y computadoras implica la postulación de símbolos mentales. De modo que no hay computación sin representación.

\section{CARÁCTER REPRESENTACIONAL DE LA MENTE}

Jerry Fodor es el representante más destacado del enfoque clásico de la ciencia cognitiva. Él caracterizó la mente desde una perspectiva naturalizada mediante varios aspectos que proporcionaron una configuración objetiva y concreta a la mente humana. Tales aspectos fueron básicamente: el carácter representacional de la mente, su mecanismo computacional y su funcionalidad, con los que confirió poder causal a la mente para generar conductas. Esos tres aspectos fueron considerados como el núcleo fundamental del desarrollo de la ciencia cognitiva en la década de 1970, que es conocida como ciencia cognitiva clásica.

Fodor afirmó que la ciencia cognitiva (de la década de 1970) se fundamentaba en dos ideas básicas: por un lado, que los estados mentales son típicamente representacionales y, por otro lado, que los procesos mentales son típicamente computacionales. En el prefacio a la edición castellana de su libro El lenguaje del pensamiento $(1975,1984)$, Fodor declaró que se propuso integrar y elaborar esas dos ideas con la finalidad de fundamentar una teoría de la mente cognitiva, considerando que las teorías razonables en psicología postulaban que la cognición es como un proceso computacional y, al mismo tiempo, sostenían que el modelo computacional de la cognición conduce por necesidad a una teoría representacional de la mente, de modo que sin representación no habría computaciones, y sin computaciones no hay modelos. Con ello, Fodor quería decir que un agente cognitivo tiene que poseer un sistema representacional que le permita pensar sobre el mundo. El representacionalismo o teoría representacional de la mente considera que los estados mentales son relaciones entre los portadores de esos estados y los símbolos mentales (o representaciones mentales). Así, las representaciones mentales que están basadas en símbolos son posibles de manipulación e interactúan unas con otras mientras se encuentran mediadas entre las entradas perceptivas y las salidas conductuales.

Fodor $(1975,1984 ; 1981 ; 1987,1994 ; 1994,1996)$ elaboró y defendió una forma de representacionalismo mediante su hipótesis del Lenguaje del pensamiento (LDP), a través de la cual sostuvo que somos capaces de conocer debido a que disponemos de un lenguaje mental, el mentalés, cuyos símbolos se combinan sistemáticamente por medio de reglas sintácticas para formar unidades más complejas, como los pensamientos. Esos símbolos mentales tienen la propiedad de ser intencionales (en la medida que versan sobre algo), por lo que dan lugar a estados que son representacionales. Así, los estados mentales heredan su intencionalidad desde las representaciones mentales que los constituyen.

En suma, de acuerdo con Fodor (1981) se puede decir que un estado representacional viene a ser un estado mental auténticamente funcional, que adopta la forma de una "actitud proposicional" que a su vez tiene una representación y expresión simbólica. De esa manera, una actitud proposicional como "creer" o "desear" o "temer" que P, es estar en una cierta relación computacional para una representación de P. 


\section{Actitudes proposicionales}

El concepto de "actitudes proposicionales" es central para caracterizar la naturaleza de la representación mental, y en relación a ello Stalnaker $(1999,2002)$ manifestó lo siguiente:

Las actitudes proposicionales son estados mentales con contenido representacional. La creencia es el ejemplo más destacado de actitud proposicional. Otros incluyen la intención, el deseo y la volición, la esperanza y el miedo, el opinar y el percibir, y las presuposiciones tácitas. Los verbos de las actitudes proposicionales expresan la relación entre un agente y algún tipo de objeto abstracto (el contenido de la actitud, el objeto denotado por una oración nominalizada). Así, una afirmación como "Alfredo cree que las pulgas tienen alas" expresa que Alfredo está en una relación de creencia con que las pulgas tienen alas. El predicado "cree que las pulgas tienen alas" expresa una propiedad que se atribuye a Alfredo (Stalnaker, 1999, 2002; pág. 144).

En general, una actitud proposicional, o cualquier estado representacional, representa al mundo como siendo de cierta manera, y el contenido de la actitud determina la forma en que el mundo es representado.

\section{Funcionalismo y la máquina de Turing}

Desde una perspectiva objetiva, el funcionalismo cobra claridad conceptual y objetiva cuando es asociado con las computadoras, o sea, con el funcionamiento de las máquinas de Turing. El estado mental es equiparado con el estado computacional de la máquina u operación que la máquina desempeña. Haselager (1997) refirió que Church propuso la tesis que afirma que todo procedimiento que es efectivamente computable puede ser ejecutado mediante un procedimiento recursivo. Y por otro lado, Turing probó que una máquina universal que lleva su nombre (computadora) computaría todas las funciones recursivas. La síntesis de esas dos ideas dio lugar a la tesis de Church-Turing, que en esencia dice: todo proceso que puede ser formalmente especificado por la vía de un procedimiento efectivo es mecánicamente ejecutable. De modo que dicha tesis concretiza el concepto de computación asociado con el mecanismo mental del pensamiento.

\section{CONCLUSIÓN}

Como conclusión cabe destacar que los símbolos presentan dos aspectos: el principal es que los símbolos son estructuras representacionales, ya que pueden versar sobre cosas; en segundo lugar, los símbolos son físicamente realizados o signalizados. Por la presencia de esas propiedades físicas, se puede afirmar que no es un misterio que los símbolos puedan tener consecuencias causales de carácter físico. Es en ese sentido que el carácter representacional de la mente cobra objetividad, concretización y poder causal para producir acciones comportamentales.

Además, considerando que los estados psicológicos sean comprendidos en términos de estados computacionales de una máquina, en la medida que los estados y procesos mentales pueden ser formalmente especificados; entonces, podemos estar seguros que ellos sean implementables mecánicamente en una máquina universal de Turing (o sea, 
en una computadora). Esas condiciones fortalecen la idea de que los estados y procesos mentales que forman parte de una explicación no sean considerados como ficciones con poderes causales inexplicables y, al mismo tiempo, constituiría una estrategia heurística para estudiar el fenómeno mental desde una perspectiva realmente científica. Por todo ello, la idea central de la teoría computacional de la mente es que la mente es un sistema de procesamiento de símbolos.

\section{REFERENCIAS BIBLIOGRÁFICAS}

1. Carnap, R. (1978). Psicología en lenguaje fisicalista (1932-33). En A. J. Ayer (Comp.), El positivismo lógico. Madrid: Fondo de Cultura Económica.

2. Churchland, P. M. (1984, 1999). Materia y conciencia. Barcelona: Ed. Gedisa.

3. Crane, T. (1992). Mental causation and mental reality. En Proceedings of the aristotelian society, 92, 185-202.

4. Crane, T. (s/d). Mental causation.

5. Davidson, D. (1991). Mental events (1970). En David M. Rosenthal (Ed.) The nature of mind. Oxford: Oxford University Press.

6. Fodor, J. A. (1994,1996). El olmo y el experto: el reino de la mente y su semántica. Barcelona: Paidós.

7. Fodor, J. A. (1987, 1994). Psicosemántica: el problema del significado en la filosofía de la mente. Madrid: Editorial Tecnos.

8. Fodor, J. A. (1981). Representations: philosophical essays on the foundations of cognitive science. Massachusetts: MIT Press.

9. Fodor, J. A. (1981). The mind-body problem. En Scientific american, 244, 1, 124-132.

10. Fodor, J. A. (1975, 1984). El lenguaje del pensamiento. Madrid: Alianza Editorial.

11. Haselager, W. F. G. (1997). Cognitive science and folk psychology: the right frame of mind. London: SAGE Publications Ltd.

12. Lycan, W. (1999, 2002). Intencionalidad. En Enciclopedia MIT de ciencias cognitivas (vol. I: A-L). Madrid: Editorial Síntesis.

13. Putnam, H. (1975). Philosophy and our mental life. En Hilary Putnam (Ed.) Philosophical papers, vol. 2. Cambridge: Cambridge University Press.

14. Rosenthal. D. M. (1991). The nature of mind. Oxford: Oxford University Press.

15. Ryle, G. (1949). The concept of mind. Harmondsworth: Peregrine.

16. Stalnaker, R. (1999, 2002). Actitudes proposicionales. En Enciclopedia MIT de ciencias cognitivas (vol. I: A-L). Madrid: Editorial Síntesis.

17. Wittgenstein, L. (1953, 1988). Investigaciones filosóficas. México: Instituto de investigaciones filosóficas, UNAM. 